\title{
Two Neighbouring Strike Slip Faults and Their Interaction
}

\author{
Papiya Debnath ${ }^{1}$, Sanjay Sen $^{2}$ \\ ${ }^{1}$ Corresponding author, Department of Basic Science and Humanities, Techno India College of Technology, \\ Rajarhat, Newtown, Kolkata-700156, India, \\ ${ }^{2}$ Department of Applied Mathematics, University of Calcutta, 92, Acharya Prafulla Chandra Road, Calcutta-
} 700009, India,

\begin{abstract}
Seismically active regions are usually associated with fault-systems comprising of a number of neighbouring faults. A movement across any one of them influences the nature of stress accumulation near the others. There may be regions of stress accumulation and/or stress release due to a movement across a fault. This interaction depends upon the relative position of the faults with respect to one another and also on the inclinations of the faults. Analytical expressions for the displacements, stresses and strains are computed using Green's function technique and correspondence principle. Numerical computation have been carried out to find out the interactions among such faults.
\end{abstract}

Keywords: Aseismic state, Correspondence Principle, Mantle convection, Seismically active regions, Strike slip fault, Viscoelastic material

\section{Introduction}

In many seismically active regions there are fault-systems consisting of a number of neighbouring faults. For example, in the western part of north America near the San Andreas fault there are a number of neighbouring faults such as Calaveras, Garlock, Hayward, San Jacinto etc. A fault movement across any one of them is likely to influence the nature of stress accumulation near the other faults. In the present paper we have considered two buried long strike slip faults situated in a half space of linear viscoelastic solid having the properties of both Maxwell and Kelvin(Voigt) type materials. Tectonic forces due to mantle convection has been assumed to be given by a slowly increasing time dependent function. These features have not been considered earlier.

\section{Formulation}

We consider two long and buried strike-slip faults $F_{1}$ and $F_{2}$ situated in a viscoelastic half space of linear viscoelastic solid material having the properties of both Maxwell and Kelvin(Voigt) type materials.

Let $d_{1}$ and $d_{2}$ are the depths of the upper edges of the faults below the free surface and $D$ is the distance measured horizontally between the upper edges of the faults. $\theta_{1}$ and $\theta_{2}$ are the inclinations of the faults with the horizontal. $D_{1}$ and $D_{2}$ are the lengths of the faults $F_{1}$ and $F_{2}$ respectively.

A set of Cartesian coordinate axes $\left(y_{1}, y_{2}, y_{3}\right)$ have been chosen as in Fig. 1 with the plane free surface $y_{3}=0$. For convenience of calculation, we introduce two more systems of Cartesian coordinates $\left(y_{1}^{\prime}, y_{2}^{\prime}, y_{3}^{\prime}\right)$, $\left(y^{\prime \prime}{ }_{1}, y^{\prime \prime}{ }_{2}, y^{\prime \prime}{ }_{3}\right)$ as shown in the figure. They are connected by the following relations:

$$
\left.\begin{array}{c}
\mathrm{y}_{1}=\mathrm{y}_{1} \\
\mathrm{y}^{\prime}{ }_{2}=\mathrm{y}_{2} \sin \theta_{1}-\left(\mathrm{y}_{3}-\mathrm{d}_{1}\right) \cos \theta_{1} \\
\mathrm{y}^{\prime}{ }_{3}=\mathrm{y}_{2} \cos \theta_{1}+\left(\mathrm{y}_{3}-\mathrm{d}_{1}\right) \sin \theta_{1} \\
\text { and } \\
\mathrm{y}^{\prime \prime}=\mathrm{z}_{1} \\
\mathrm{y}^{\prime \prime}{ }_{2}=\mathrm{z}_{2} \sin \theta_{2}-\mathrm{z}_{3} \cos \theta_{2} \\
\mathrm{y}^{\prime \prime}{ }_{3}=\mathrm{z}_{2} \cos \theta_{2}+\mathrm{z}_{3} \sin \theta_{2}
\end{array}\right\}
$$

For long fault the displacements, stresses and strains are assumed to be independent of $y_{1}$ and depended on $\mathrm{y}_{2}, \mathrm{y}_{3}$ and time t. This separates out the displacements, stresses and strains into two independent groups: one group containing $\mathrm{u}, \tau_{12}, \tau_{13}, \mathrm{e}_{12}$ and $\mathrm{e}_{13}$ associated with strike slip movement. The remaining components are associated with a possible dip slip movement of the fault. We consider here the strike slip movement across the faults.

We take $\mathrm{t}=0$ at an instant when the medium is in aseismic state.

The stress-strain relationship can be taken as: 


$$
\left.\begin{array}{c}
\tau_{12}+\frac{\eta}{\mu} \frac{\partial}{\partial \mathrm{t}}\left(\tau_{12}\right)=\mu \frac{\partial \mathrm{u}}{\partial \mathrm{y}_{2}}+2 \eta \frac{\partial}{\partial \mathrm{t}}\left(\frac{\partial \mathrm{u}}{\partial \mathrm{y}_{2}}\right) \\
\tau_{13}+\frac{\eta}{\mu} \frac{\partial}{\partial \mathrm{t}}\left(\tau_{13}\right)=\mu \frac{\partial \mathrm{u}}{\partial \mathrm{y}_{3}}+2 \eta \frac{\partial}{\partial \mathrm{t}}\left(\frac{\partial \mathrm{u}}{\partial \mathrm{y}_{3}}\right)
\end{array}\right\}
$$

where $\eta$ is the effective viscosity and $\mu$ is the effective rigidity of the material.

The stresses satisfy the following equation of motion :

$$
\left.\begin{array}{c}
\frac{\partial}{\partial \mathrm{y}_{2}}\left(\tau_{12}\right)+\frac{\partial}{\partial \mathrm{y}_{3}}\left(\tau_{13}\right)=0 \\
\left(-\infty<\mathrm{y}_{2}<\infty, \mathrm{y}_{3} \geq 0, \mathrm{t} \geq 0\right)
\end{array}\right\}
$$

[Assuming that the external forces do not change significantly during our investigation]

We consider aseismic state of the model during which the inertial terms are very small and are neglected in the above equation.

The boundary conditions are:

$$
\left.\begin{array}{c}
\tau_{13}=0 \text { on } \mathrm{y}_{3}=0,\left(-\infty<\mathrm{y}_{2}<\infty, \quad t \geq 0\right) \\
\tau_{13} \rightarrow 0 \text { as } \mathrm{y}_{3} \rightarrow \infty, \quad\left(-\infty<\mathrm{y}_{2}<\infty, \quad t \geq 0\right)
\end{array}\right\}
$$

Mantle convection introduces tectonic forces in the lithosphere-asthenosphere system far away from the faults which causes the faults to slip leading to an earthquake. We represent these tectonic forces by $\tau_{\infty}(t)$ and assume it to be a slowly increasing function of time and write

$\tau_{\infty}(t)=\tau_{\infty}(0)(1+k t)$, where $k>0$

Then, the relevant boundary conditions become:

$$
\left.\begin{array}{c}
\tau_{12} \rightarrow \tau_{\infty}(t)=\tau_{\infty}(0)(1+k t), \\
(k>0) \text { as }\left|y_{2}\right| \rightarrow \infty \text {, for } y_{3} \geq 0, t \geq 0 .
\end{array}\right\}
$$

Now differentiating partially equation (2) with respect to $y_{2}$ and with respect to $y_{3}$ and adding them using equation (3) we get,

$\nabla^{2} \mathrm{u}\left(\mathrm{y}_{2}, \mathrm{y}_{3}, \mathrm{t}\right)=\mathrm{c} \cdot \mathrm{e}^{-\frac{\mu \mathrm{t}}{2 \eta}},(\mathrm{c}$, an arbitrary constant $)$

and $\nabla^{2} \mathrm{U}=0$

where, $U=u-(u)_{0} e^{-\frac{\mu t}{2 \eta}}$

We assume that $(u)_{0},\left(\tau_{12}\right)_{0},\left(\tau_{13}\right)_{0},\left(e_{12}\right)_{0}$ and $\left(e_{13}\right)_{0}$ are the values of $u, \tau_{12}, \tau_{13}, e_{12}$ and $e_{13}$ respectively at time $\mathrm{t}=0$. They are functions of $\mathrm{y}_{2}, \mathrm{y}_{3}$ and satisfy the relations (2) to (4c).

\section{Displacements, Stresses and Strains in the Absence of any Fault Movement}

The above boundary value problem given by (2) to (5) has been solved by taking Laplace transform with respect to time $t$ of all constitutive equations and boundary conditions. Taking the inverse Laplace transform the solutions are obtained as:

$$
\begin{aligned}
& \left.\mathrm{u}=(\mathrm{u})_{0} \mathrm{e}^{-\frac{\mu \mathrm{t}}{2 \eta}}+\mathrm{y}_{2} \tau_{\infty}(0)\left[\frac{1}{\mu}-\frac{\eta \mathrm{k}}{\mu^{2}}+\frac{\mathrm{kt}}{\mu}+\left(\frac{\eta \mathrm{k}}{\mu^{2}}-\frac{1}{\mu}\right) \mathrm{e}^{-\frac{\mu \mathrm{t}}{2 \eta}}\right]\right) \\
& \mathrm{e}_{12}=\left(\mathrm{e}_{12}\right)_{0} \mathrm{e}^{-\frac{\mu \mathrm{t}}{2 \eta}}+\tau_{\infty}(0)\left[\frac{1}{\mu}-\frac{\eta \mathrm{k}}{\mu^{2}}+\frac{\mathrm{kt}}{\mu}+\left(\frac{\eta \mathrm{k}}{\mu^{2}}-\frac{1}{\mu}\right) \mathrm{e}^{-\frac{\mu \mathrm{t}}{2 \eta}}\right] \\
& \tau_{12}=\tau_{\infty}(0)\left(1+\mathrm{kt}-\mathrm{e}^{-\frac{\mu \mathrm{t}}{\eta}}\right)++\left(\tau_{12}\right)_{0} \mathrm{e}^{-\frac{\mu \mathrm{t}}{\eta}} \\
& \tau_{13}=\left(\tau_{13}\right)_{0} \mathrm{e}^{-\frac{\mu t}{\eta}} \\
& \tau_{1^{\prime} 2^{\prime}}=\text { The stress across the fault } \mathrm{F}_{1} \\
& =\tau_{12} \sin \theta_{1}-\tau_{13} \cos \theta_{1} \\
& \left.=\left(\tau_{1}^{\prime} 2^{\prime}\right)_{0} \mathrm{e}^{-\frac{\mu \mathrm{t}}{\eta}}+\tau_{\infty}(0)\left(1+\mathrm{kt}-\mathrm{e}^{-\frac{\mu \mathrm{t}}{\eta}}\right) \sin \theta_{1}, \quad \mathrm{t} \geq 0\right)
\end{aligned}
$$

where $\left(\tau_{1}{ }^{\prime} 2^{\prime}\right)_{0}$ is the value of $\tau_{1}{ }^{\prime} 2^{\prime}$ at $t=0$ which is a function of $y_{2}, y_{3}$.

Similar equation for the fault $\mathrm{F}_{2}$ is given by:

$\tau_{1 "}{ }^{\prime \prime}{ }^{\prime \prime}=$ The stress across the fault $F_{2}$

$=\tau_{12} \sin \theta_{2}-\tau_{13} \cos \theta_{2}$

We observed that for the fault $F_{1}$ the relevent stress component $\tau_{1}{ }^{\prime} 2^{\prime}$ increases with time and finally tends to $\tau_{\infty}(t) \sin \theta_{1}$ but the rheological nature of the region in the neighbourhood of $F_{1}$ has been assumed to be such that it slips when the magnitude of stress $\tau_{1^{\prime} 2^{\prime}}$ reaches a threshold value say $\left(\tau_{c}\right)_{1}\left(<\tau_{\infty}(t) \sin \theta_{1}\right)$ after a time, say $\mathrm{T}_{1}$. 
Similarly, for the fault $F_{2}$ when the stress $\tau_{1^{\prime \prime} 2^{\prime \prime}}$ exceeds the critical value say $\left(\tau_{c}\right)_{2}\left(<\tau_{\infty}(t) \sin \theta_{2}\right) F_{2}$ slips after a time say $T_{2}$. We assume that $\left(\tau_{c}\right)_{1}<\left(\tau_{c}\right)_{2}$ so that the fault $F_{1}$ slips first before $F_{2}$.

The slip across $F_{1}$ generates seismic waves in the system which gradually die out with the passage of time and aseismic state re-established in the system. During this small interval of time when the seismic disturbances were present in the system, our stress equations of motion (3) were not valid. We leave out this small amount of time (of the order of few minutes at the most) and consider our model afresh when aseismic state re-established and set up our new time origin $t=0$ suitably. Due to the slip across $\mathrm{F}_{1}$ a considerable part of the accumulated stress near it got released. Observation shows that in major earthquakes more than 80 percent of the accumulated stress released through a slipping movement across the fault.

\section{Displacements, Stresses and Strains After the Commencement of the Fault Movement}

We assume that the accumulated stress near $F_{1}$ came down from the critical level $\left(\tau_{c}\right)_{1}$ to a level $\left(\tau_{1}{ }^{\prime}{ }^{\prime}\right)_{p}$. All the basic equations (2) to (5) remain valid for the second phase of our model. But now we have an additional dislocation condition across $\mathrm{F}_{1}$ given by:

$[\mathrm{u}]_{\mathrm{F}_{1}}=\mathrm{U}_{1} \mathrm{f}_{1}\left(\mathrm{y}_{3}{ }^{\prime}\right) \mathrm{H}\left(\mathrm{t}_{1}\right)$ across $\mathrm{F}_{1}$.

$$
\left(\mathrm{y}_{2}^{\prime}=0, \quad 0 \leq \mathrm{y}_{3}^{\prime} \leq \mathrm{D}_{1}, \mathrm{t}_{1}=\mathrm{t}-\mathrm{T}_{1}\right)
$$

where $[\mathrm{u}]$ is the discontinuity in $\mathrm{u}$ across $\mathrm{F}_{1}$, and $\mathrm{H}\left(\mathrm{t}_{1}\right)$ is Heaviside unit step function.

We solved the resulting boundary value problem by modified Green's function method following [1],

[2] and correspondence principle (as shown in Appendix) and get the final solution for Displacement, Strain and Stresses after the movement across $\mathrm{F}_{2}\left(\mathrm{t}-\mathrm{T}_{2}>0\right)$ as:

$$
\left.\begin{array}{c}
\mathrm{u}=(\mathrm{u})_{\mathrm{p}} \mathrm{e}^{-\frac{\mu \mathrm{t}}{2 \eta}}+\mathrm{y}_{2} \tau_{\infty}(0)\left[\frac{1}{\mu}-\frac{\eta \mathrm{k}}{\mu^{2}}+\frac{\mathrm{kt}}{\mu}+\left(\frac{\eta \mathrm{k}}{\mu^{2}}-\frac{1}{\mu}\right) \mathrm{e}^{-\frac{\mu \mathrm{t}}{2 \eta}}\right] \\
+\frac{\mathrm{U}_{1}}{2 \pi} \mathrm{H}\left(\mathrm{t}-\mathrm{T}_{1}\right) \Psi_{1}\left(\mathrm{y}_{2}, \mathrm{y}_{3}\right)+\frac{\mathrm{U}_{2}}{2 \pi} \mathrm{H}\left(\mathrm{t}-\mathrm{T}_{2}\right) \phi_{1}\left(\mathrm{y}_{2}, \mathrm{y}_{3}\right) \\
\mathrm{e}_{12}=\left(\mathrm{e}_{12}\right)_{\mathrm{p}} \mathrm{e}^{-\frac{\mu \mathrm{t}}{2 \eta}}+\tau_{\infty}(0)\left[\frac{1}{\mu}-\frac{\eta \mathrm{k}}{\mu^{2}}+\frac{\mathrm{kt}}{\mu}+\left(\frac{\eta \mathrm{k}}{\mu^{2}}-\frac{1}{\mu}\right) \mathrm{e}^{-\frac{\mu \mathrm{t}}{2 \eta}}\right] \\
+\frac{\mathrm{U}_{1}}{2 \pi} \mathrm{H}\left(\mathrm{t}-\mathrm{T}_{1}\right) \Psi_{2}\left(\mathrm{y}_{2}, \mathrm{y}_{3}\right)+\frac{\mathrm{U}_{2}}{2 \pi} \mathrm{H}\left(\mathrm{t}-\mathrm{T}_{2}\right) \phi_{2}\left(\mathrm{y}_{2}, \mathrm{y}_{3}\right) \\
\tau_{12}=\left(\tau_{12}\right)_{\mathrm{p}} \mathrm{e}^{-\frac{\mu \mathrm{t}}{\eta}}+\tau_{\infty}(0)\left(1+\mathrm{kt}-\mathrm{e}^{-\frac{\mu \mathrm{t}}{\eta}}\right) \\
+\frac{\mu \mathrm{U}_{1}}{2 \pi} \mathrm{H}\left(\mathrm{t}-\mathrm{T}_{1}\right)\left(1+\mathrm{e}^{-\frac{\mu \mathrm{t}}{\eta}}\right) \Psi_{2}\left(\mathrm{y}_{2}, \mathrm{y}_{3}\right)+\frac{\mu \mathrm{U}_{2}}{2 \pi} \mathrm{H}\left(\mathrm{t}-\mathrm{T}_{2}\right)\left(1+\mathrm{e}^{-\frac{\mu \mathrm{t}}{\eta}}\right) \phi_{2}\left(\mathrm{y}_{2}, \mathrm{y}_{3}\right) \\
\left.\tau_{13} \tau_{13}\right)_{\mathrm{p}} \mathrm{e}^{-\frac{\mu \mathrm{t}}{\eta}}+\frac{\mu \mathrm{U}_{1}}{2 \pi} \mathrm{H}\left(\mathrm{t}-\mathrm{T}_{1}\right)\left(1+\mathrm{e}^{-\frac{\mu \mathrm{t}}{\eta}}\right) \Psi_{3}\left(\mathrm{y}_{2}, \mathrm{y}_{3}\right)+\frac{\mu \mathrm{U}_{2}}{2 \pi} \mathrm{H}\left(\mathrm{t}-\mathrm{T}_{2}\right)\left(1+\mathrm{e}^{-\frac{\mu \mathrm{t}}{\eta}}\right) \phi_{3}\left(\mathrm{y}_{2}, \mathrm{y}_{3}\right) \\
\tau_{1}^{\prime \prime} 2^{\prime \prime}=\left(\tau_{1}^{\prime \prime} 2^{\prime \prime}\right)_{\mathrm{p}} \mathrm{e}^{-\frac{\mu \mathrm{t}}{\eta}}+\tau_{\infty}(0)\left(1+\mathrm{kt}-\mathrm{e}^{-\frac{\mu \mathrm{t}}{\eta}}\right) \sin \theta_{2} \\
+\frac{\mu \mathrm{U}_{1}}{2 \pi} \mathrm{H}\left(\mathrm{t}-\mathrm{T}_{1}\right)\left(1+\mathrm{e}^{-\frac{\mu \mathrm{t}}{\eta}}\right)\left[\Psi_{2}\left(\mathrm{y}_{2}, \mathrm{y}_{3}\right) \sin \theta_{1}-\psi_{3}\left(\mathrm{y}_{2}, \mathrm{y}_{3}\right) \cos \theta_{1}\right] \\
+\frac{\mu \mathrm{U}_{2}}{2 \pi} \mathrm{H}\left(\mathrm{t}-\mathrm{T}_{2}\right)\left(1+\mathrm{e}^{-\frac{\mu \mathrm{t}}{\eta}}\right)\left[\phi_{2}\left(\mathrm{y}_{2}, \mathrm{y}_{3}\right) \sin \theta_{2}-\phi_{3}\left(\mathrm{y}_{2}, \mathrm{y}_{3}\right) \cos \theta_{2}\right]
\end{array}\right\}
$$

where, $\psi_{1}, \psi_{2}, \psi_{3}$ and $\phi_{1}, \phi_{2}, \phi_{3}$ are given in the Appendix.

It has been observed, as in [3] that the strains and the stresses will remain bounded everywhere in the model, including the upper and lower edges of the faults, the functions $f_{1}$ and $f_{2}$ should satisfy the following sufficient conditions:

(I) $f\left(y_{3}\right), f^{\prime}\left(y_{3}\right)$ are continuous in $0 \leq y_{3} \leq D_{1}$,

II) Either (a) $f^{\prime \prime}\left(y_{3}\right)$ is continuous in $0 \leq y_{3} \leq D_{1}$,

or (b) $f^{\prime \prime}\left(y_{3}\right)$ is continuous in $0 \leq y_{3} \leq D_{1}$, except for a finite number of points of finite discontinuity in $0 \leq y_{3} \leq D_{1}$,

or (c) $f^{\prime \prime}\left(y_{3}\right)$ is continuous in $0 \leq y_{3} \leq D_{1}$, except possibly for a finite number of points of finite discontinuity and for the ends points of $\left(0, D_{1}\right)$, there exist real constants $m<1$ and $n<1$ such that $y_{3}{ }^{m} f^{\prime \prime}\left(y_{3}\right) \rightarrow 0$ or to a finite limit as $y_{3} \rightarrow 0+0$ and $\left(D_{1}-y_{3}\right)^{n} f^{\prime \prime}\left(y_{3}\right) \rightarrow 0$ or to a finite limit as $y_{3} \rightarrow D_{1}-0$ and

(III) $f\left(D_{1}\right)=0=f^{\prime}\left(D_{1}\right), \quad f^{\prime}(0)=0$,

These are sufficient conditions which ensure finite displacements, stresses and strains for all finite $\left(y_{2}, y_{3}, t\right)$.

We can evaluate the integrals if $f\left(y_{3}\right)$ is any polynomial satisfying (I),(II) and (III). One such function is

$$
f\left(y^{\prime}{ }_{3}\right)=\frac{{y^{\prime}}_{3}{ }^{2}\left(y_{3}^{\prime}-D_{1}\right)^{2}}{\left(\frac{D_{1}}{2}\right)^{4}}
$$


We consider $f_{1}\left(\xi^{\prime}\right)$ to be

\section{Numerical Computations}

$$
f_{1}\left(\xi_{3}^{\prime}\right)=\frac{\xi_{3}^{\prime}{ }_{3}^{2}\left(\xi_{3}^{\prime}-D_{1}\right)^{2}}{\left(\frac{D_{1}}{2}\right)^{4}}
$$

(and a similar function for $f_{2}\left(\eta_{3}^{\prime}\right)$ ) which satisfies all the conditions for bounded strains and stresses stated above.

Following [4], [5] and the recent studies on rheological behaviour of crust and upper mantle by [6], [7] the values to the model parameters are taken as:

$\mu=3.5 \times 10^{11}$ dyne $/$ sq. $\mathrm{cm}$.

$\eta=5 \times 10^{20}$ poise

$d_{1}$ and $d_{2}=$ Depths of the faults $F_{1}$ and $F_{2}$ below the free surface $=10 \mathrm{~km}$. and $25 \mathrm{~km}$. respectively ( noting that the depth of the major earthquake faults are in between $10-30 \mathrm{~km}$.)

$t_{1}=t-T_{1}$

$t_{2}=t-T_{2}$

$\tau_{\infty}(t)=\tau_{\infty}(0)(1+k t), \quad k=10^{-9}$

$\tau_{\infty}(0)=50 \mathrm{bar}$

$\left(\tau_{12}\right)_{0}=50$ bar

$\left(\tau_{13}\right)_{0}=50 \mathrm{bar}$

$\left(\tau_{c}\right)_{1}=200$ bar

$\left(\tau_{c}\right)_{2}=250$ bar

$\mathrm{D}=10 \mathrm{~km} .=$ Distance measure along the horizontal axes between the upper edges of the fault.

\section{Discussion of the Results}

In regions near California observations indicate that the rheological behaviour of the materials are such that the frictional and cohesive forces across the faults can withstand stresses of the order of 400 bars before slipping. In the present case, we assume that $\left(\tau_{c}\right)_{1} \approx 200$ bar and $\left(\tau_{c}\right)_{2} \approx 250$ bar. The computed times $T_{1}$ and $T_{2}$ for slips across vertical faults $F_{1}$ and $F_{2}$ to occur are found to be $T_{1}$ approx 98 years and $T_{2} \approx$ 160 respectively. For inclined faults, $T_{1} \approx 149$ and 117 years for $\theta_{1}=\frac{\pi}{4}$ and $\frac{\pi}{3}$ respectively, and $T_{2}=193$ and 152 years for $\theta_{2}=\frac{\pi}{4}$ and $\frac{\pi}{3}$ respectively. We assume that a major earthquake occurs due to the slip across $F_{1}$ and 80 percent of the accumulated stress released, so that $\left(\tau_{1}{ }^{\prime} 2^{\prime}\right)_{p} \approx 40$ bar in the expression (7).

We compute:

\subsection{The effect of fault-slip across $F_{1}$ on the stress accumulation at a point near the middle of the} neighbouring fault $\boldsymbol{F}_{2}$

The effect of fault-slip across $F_{1}$ on the stress accumulation at a point near the middle of the neighbouring fault $F_{2}$ given by:

$$
\frac{\mu U_{1}}{2 \pi} H\left(t-T_{1}\right)\left(1+e^{-\frac{\mu t}{\eta}}\right)\left[\psi_{2}\left(y_{2}, y_{3}\right) \sin \theta_{1}-\psi_{3}\left(y_{2}, y_{3}\right) \cos \theta_{1}\right] \text { at } y_{2}^{\prime \prime} \approx 0.5 \mathrm{~km} \text { and } y_{3}^{\prime \prime} \approx 5 \mathrm{~km}
$$

This has been shown in Fig. (2) $\left(\theta_{1}=\frac{\pi}{4}\right.$ and $\left.\theta_{2}=\frac{\pi}{4}\right)$ we find that the magnitude of the stress $\tau_{1^{\prime} 2^{\prime}}$ near the mid point of $F_{2}$ is the order of 4.3 bar. Initially, just after $T_{1}$ its value was little more than 4.3 bar which came down slowly to 4.1 bar after a laps of about 400 years. It retains almost the same value but having a decreasing trend. The positive sign indicate that there is an increase in stress accumulation near $F_{2}$ due to a fault slip across $F_{1}$.

\subsection{The effect of fault-slip across $F_{2}$ on the stress accumulation at a point near the middle of the} neighbouring fault $\boldsymbol{F}_{1}$

The effect of fault-slip across $F_{2}$ on the stress accumulation at a point near the middle of the neighbouring fault $F_{1}$ given by:

$$
\frac{\mu U_{2}}{2 \pi} H\left(t-T_{2}\right)\left(1+e^{-\frac{\mu t}{\eta}}\right)\left[\phi_{2}\left(y_{2}, y_{3}\right) \sin \theta_{2}-\phi_{3}\left(y_{2}, y_{3}\right) \cos \theta_{2}\right] \text { at } y_{2}{ }^{\prime} \approx 0.5 \mathrm{~km} \text { and } y_{3}{ }^{\prime} \approx 5 \mathrm{~km}
$$

From Fig. (3) we find that the magnitude of the stress $\tau_{1 "}{ }^{\prime \prime}$ " near the midpoint of the fault $F_{1}$ has a value 2.38 bar initially which decreases to a value 2.35 bar after a laps of about 400 years.

\subsection{Stress accumulation against time at a point $y_{2}=8 \mathrm{~km} ., y_{3}=8 \mathrm{~km}$. After the movement of the faults}

Fig. (4) shows that the pattern of total stress over time at a particular point given by $y_{2}=8 \mathrm{~km}$., $y_{3}=8$ $\mathrm{km}$. Total stress accumulation depends upon the inclination of the fault. Sudden release of stress at $T_{1}$ and $T_{2}$ are clearly visible. The stress increases at a slightly decreasing rate over years. 


\subsection{Region of stress accumulation and release}

Fig. (5a) shows the regions of stress accumulation and release due to the fault movement across $F_{1}$ only (with fault $F_{1}$ is shown in black colour).

Fig. (5b) shows the regions of stress accumulation and release after the slip across both the faults $F_{1}$ and $F_{2}$ (with faults $F_{1}$ and $F_{2}$ are shown in black colours).

\subsection{Contour map}

Fig. (6a) shows contour map for stress accumulation/release in the medium due to the fault slip across $F_{1}$ (with fault $F_{1}$ is shown in black colour).

Fig. (6b) shows contour map for stress accumulation/release in the medium due to the fault slip across both the faults $F_{1}$ and $F_{2}$ (with faults $F_{1}$ and $F_{2}$ are shown in black colours).

\section{Appendix}

\subsection{Displacements, stresses, and strains before the commencement of the fault movement}

We take Laplace transform of all constitutive equations and boundary conditions

$\overline{\tau_{12}}=\frac{\frac{\partial \bar{u}}{\partial y_{2}}(\mu+2 \eta p)}{1+\frac{\eta p}{\mu}}+\frac{\frac{\eta}{\mu}\left(\tau_{12}\right)_{0}}{1+\frac{\eta p}{\mu}}-\frac{2 \eta\left(\frac{\partial u}{\partial y_{2}}\right)_{0}}{1+\frac{\eta p}{\mu}}$

where $\overline{\tau_{12}}=\int_{0}^{\infty} \tau_{12} e^{-p t_{1}} d t$, p being the Laplace transform variable.

and a similar equation for $\overline{\tau_{13}}$.

Also the stress equation of motion in Laplace transform domain as:

$\frac{\partial}{\partial y_{2}}\left(\overline{\tau_{12}}\right)+\frac{\partial}{\partial y_{3}}\left(\overline{\tau_{13}}\right)=0$

and the boundary conditions are:

$$
\left.\begin{array}{l}
\overline{\tau_{13}}=0 \text { on } y_{3}=0,\left(-\infty<y_{2}<\infty, \quad t_{1} \geq 0\right) \\
\rightarrow 0 \text { as } y_{3} \rightarrow \infty, \quad\left(-\infty<y_{2}<\infty, \quad t_{1} \geq 0\right)
\end{array}\right\}
$$

$\overline{\tau_{12}} \rightarrow \overline{\tau_{\infty}}(p)$ as $\left|y_{2}\right| \rightarrow \infty$, for $y_{2} \geq 0, t_{1} \geq 0$.

Using (8) and other similar equation, we get from (9)

$$
\nabla^{2} \bar{U}=0
$$

Thus we are to solve the boundary value problem (12) with the boundary conditions (10) to (11) Let,

be the solution of (12), where

$$
\bar{u}=\frac{(u)_{0}}{p+\frac{\mu}{2 \eta}}+A y_{2}+B y_{3}
$$

$$
\bar{U}=\bar{u}-\frac{(u)_{0}}{p+\frac{\mu}{2 \eta}}
$$

Using the boundary conditions (10) to (11) and the initial conditions we get,

$$
\begin{gathered}
A=\frac{\mu+\eta p}{\mu(\mu+2 \eta p)} \overline{\tau_{\infty}}(p)-\frac{\eta \tau_{\infty}(0)}{\mu(\mu+2 \eta p)} \\
B=0
\end{gathered}
$$

On taking inverse Laplace transform, we get

$$
\begin{gathered}
u=(u)_{0} e^{-\frac{\mu t}{2 \eta}}+y_{2} \tau_{\infty}(0)\left[\frac{1}{\mu}-\frac{\eta k}{\mu^{2}}+\frac{k t}{\mu}+\left(\frac{\eta k}{\mu^{2}}-\frac{1}{\mu}\right) e^{-\frac{\mu t}{2 \eta}}\right] \\
e_{12}=\left(e_{12}\right)_{0} e^{-\frac{\mu t}{2 \eta}}+\tau_{\infty}(0)\left[\frac{1}{\mu}-\frac{\eta k}{\mu^{2}}+\frac{k t}{\mu}+\left(\frac{\eta k}{\mu^{2}}-\frac{1}{\mu}\right) e^{-\frac{\mu t}{2 \eta}}\right] \\
\tau_{12}=\tau_{\infty}(0)\left(1+k t-e^{-\frac{\mu t}{\eta}}\right)+\left(\tau_{12}\right)_{0} e^{-\frac{\mu t}{\eta}} \\
\tau_{13}=\left(\tau_{13}\right)_{0} e^{-\frac{\mu t}{\eta}} \\
\left(\tau_{1^{\prime} 2^{\prime}}\right)_{1}=\left(\tau_{1^{\prime} 2^{\prime}}\right)_{0} e^{-\frac{\mu t}{\eta}}+\tau_{\infty}(0)\left(1+k t-e^{-\frac{\mu t}{\eta}}\right) \sin \theta_{1}
\end{gathered}
$$




\subsection{Displacements, stresses and strains after the fault slip}

We assume a gradual accumulation of shear stress near the faults which ultimately results in a movement across the faults. We now consider a sudden movement across any one of the faults, say $F_{1}$, commencing at a time $\mathrm{t}=T_{1},\left(T_{1}>0\right)$ while the other (here $F_{2}$ ) remains locked since $\left(\tau_{c}\right)_{1}<\left(\tau_{c}\right)_{2}$. Afterwards at time $\mathrm{t}=T_{2},\left(\geq T_{1}>0\right)$, the second fault $F_{2}$ also undergoes sudden movement. All the equations (2) to (5) remain valid for $\mathrm{t} \geq T_{1}$ also, but in addition we have the following conditions which characterise the sudden movement across $F_{1}$ and $F_{2}$ :

$$
[u]_{F_{1}}=U_{1} f_{1}\left(y_{3}^{\prime}\right) H\left(t_{1}\right) \text { across } F_{1} .\left(y_{2}^{\prime}=0, \quad 0 \leq y_{3}^{\prime} \leq D_{1}, t_{1}=t-T_{1} \geq 0\right)
$$

$[u]_{F_{2}}=U_{2} f_{2}\left(y_{3}^{\prime \prime}\right) H\left(t_{2}\right)$ across $\left.F_{2} .\left(y_{2}{ }^{\prime \prime}=0, \quad 0 \leq y_{3}^{\prime \prime} \leq D_{2}, t_{2}=t-T_{2} \geq 0\right)\right\}$

where $U_{1}$ and $U_{2}$ are the dislocations across $F_{1}$ and $F_{2}$ respectively. The functions $f_{1}\left(y_{3}{ }^{\prime}\right)$ and $f_{2}\left(y_{3}{ }^{\prime}\right)$ represent the depth-dependence of the dislocations along the faults $F_{1}$ and $F_{2} .[u]_{F_{1}}$ and $[u]_{F_{2}}$ are the relative displacements acorss $F_{1}$ and $F_{2}$ respectively and $H\left(t_{1}\right), H\left(t_{2}\right)$ are the Heaviside unit step functions.

We try to obtain the solutions for $\mathrm{u}, e_{12}, \tau_{12}$ and $\tau_{13}$ in the following form:

$$
\left.\begin{array}{c}
u=(u)_{1}+(u)_{2}+(u)_{3} \\
e_{12}=\left(e_{12}\right)_{1}+\left(e_{12}\right)_{2}+\left(e_{12}\right)_{3} \\
\tau_{12}=\left(\tau_{12}\right)_{1}+\left(\tau_{12}\right)_{2}+\left(\tau_{12}\right)_{3} \\
\tau_{13}=\left(\tau_{13}\right)_{1}+\left(\tau_{13}\right)_{2}++\left(\tau_{13}\right)_{3}
\end{array}\right\}
$$

where $(u)_{1},\left(e_{12}\right)_{1},\left(\tau_{12}\right)_{1}$ and $\left(\tau_{13}\right)_{1}$ satisfy the relations (2) to (5) and are similar to the displacements, stresses and strain in the absence of any fault movement. They are given by

$$
\begin{gathered}
(u)_{1}=(u)_{p} e^{-\frac{\mu t}{2 \eta}}+y_{2} \tau_{\infty}(0)\left[\frac{1}{\mu}-\frac{\eta k}{\mu^{2}}+\frac{k t}{\mu}+\left(\frac{\eta k}{\mu^{2}}-\frac{1}{\mu}\right) e^{-\frac{\mu t}{2 \eta}}\right] \\
\left(e_{12}\right)_{1}=\left(e_{12}\right)_{p} e^{-\frac{\mu t}{2 \eta}}+\tau_{\infty}(0)\left[\frac{1}{\mu}-\frac{\eta k}{\mu^{2}}+\frac{k t}{\mu}+\left(\frac{\eta k}{\mu^{2}}-\frac{1}{\mu}\right) e^{-\frac{\mu t}{2 \eta}}\right] \\
\left(\tau_{12}\right)_{1}=\left(\tau_{12}\right)_{p} e^{-\frac{\mu t}{\eta}}+\tau_{\infty}(0)\left(1+k t-e^{-\frac{\mu t}{\eta}}\right) \\
\tau_{13}=\left(\tau_{13}\right)_{p} e^{-\frac{\mu t}{\eta}} \\
\left(\tau_{1^{\prime} 2^{\prime}}\right)_{1}=\left(\tau_{1^{\prime} 2^{\prime}}\right)_{p} e^{-\frac{\mu t}{\eta}}+\tau_{\infty}(0)\left(1+k t-e^{-\frac{\mu t}{\eta}}\right) \sin \theta_{1}
\end{gathered}
$$

where $(u)_{p},\left(e_{12}\right)_{p},\left(\tau_{12}\right)_{p},\left(\tau_{13}\right)_{p},\left(\tau_{1^{\prime} 2^{\prime}}\right)_{p}$ are the initial values of $u, e_{12}, \tau_{12}, \tau_{13}$ and $\tau_{1^{\prime} 2^{\prime}}$ with respect to the new time origin.

This part of the solution represents the effect of initial field ( with respect to the new time origin) and of the tectonic forces due to mantle convection.

Now $(u)_{2},\left(e_{12}\right)_{2},\left(\tau_{12}\right)_{2},\left(\tau_{13}\right)_{2}$ are all zeros for $t \leq T_{1}$ and satisfy the relation (2) to (5) and (13) together with the following condition

$$
\left(\tau_{12}\right)_{2} \rightarrow 0 \text { as }\left|y_{2}\right| \rightarrow \infty \quad y_{3} \geq 0, t_{1} \geq 0 \text {, replacing (4b). }
$$

and $(u)_{3},\left(e_{12}\right)_{3},\left(\tau_{12}\right)_{3},\left(\tau_{13}\right)_{3}$ are all zeros for $t \leq T_{2}$ and satisfy the relation (2) to (5) and (13) together with the following condition

$$
\left(\tau_{12}\right)_{3} \rightarrow 0 \text { as }\left|z_{2}\right| \rightarrow \infty \quad z_{3} \geq 0, t_{2} \geq 0 \text {, replacing (4b) }
$$

We now consider the boundary value problem for $(u)_{2},\left(\tau_{12}\right)_{2},\left(\tau_{13}\right)_{2}$ which are the functions of $y_{2}, y_{3}$ and time $t$, satisfy the equations (2) to (5) and (13). This part represents the effect of fault slip across $F_{1}$ on the system. To obtain the solutions for $(u)_{2},\left(\tau_{12}\right)_{2},\left(\tau_{13}\right)_{2}$ we take Laplace transforms with respect to $t_{1}\left(=t-T_{1}\right)$ and obtain a boundary value problem involving $(\bar{u})_{2},\left(\overline{\tau_{12}}\right)_{2},\left(\overline{\tau_{13}}\right)_{2}$ which are the Laplace transforms of $(u)_{2},\left(\tau_{12}\right)_{2},\left(\tau_{13}\right)_{2}$ respectively with respect to $t_{1}$.

Therefore,

$$
\left\{(\bar{u})_{2},(\bar{u})_{3},\left(\overline{\tau_{12}}\right)_{2},\left(\overline{\tau_{12}}\right)_{3},\left(\overline{\tau_{13}}\right)_{2},\left(\overline{\tau_{13}}\right)_{3}\right\}=\int_{0}^{\infty}\left\{(u)_{2},(u)_{3},\left(\tau_{12}\right)_{2},\left(\tau_{12}\right)_{3},\left(\tau_{13}\right)_{2},\left(\tau_{13}\right)_{3}\right\} e^{-p t_{1}} d t_{1}
$$

where $\mathrm{p}$ is the Laplace transform variable.

We have the following relations in transformed domain

$$
\left(\overline{\tau_{12}}\right)_{2}=\frac{(\mu+2 \eta p)}{1+\frac{\eta p}{\mu}} \frac{\partial(\bar{u})_{2}}{\partial y_{2}}
$$




$$
\begin{aligned}
& \left(\overline{\tau_{13}}\right)_{2}=\frac{(\mu+2 \eta p)}{1+\frac{\eta p}{\mu}} \frac{\partial(\bar{u})_{2}}{\partial y_{3}} \\
& \frac{\partial}{\partial y_{2}}\left(\overline{\tau_{12}}\right)_{2}+\frac{\partial}{\partial y_{3}}\left(\overline{\tau_{13}}\right)_{2}=0 \\
& \nabla^{2}(\bar{u})_{2}=0 \\
& \left(\overline{\tau_{13}}\right)_{2}=0 \text { on } y_{3}=0, \quad\left(-\infty<y_{2}<\infty, \quad t \geq 0\right) \\
& \left(\overline{\tau_{13}}\right)_{2} \rightarrow 0 \text { as } y_{3} \rightarrow \infty, \quad\left(-\infty<y_{2}<\infty, \quad t \geq 0\right) \\
& \left(\overline{\tau_{12}}\right)_{2} \rightarrow 0 \text { as }\left|y_{2}\right| \rightarrow \infty, \text { for } y_{2} \geq 0, t \geq 0 . \\
& {\left[(\bar{u})_{2}\right]_{F_{1}}=\frac{U_{1}}{p} f_{1}\left(y_{3}^{\prime}\right) \text { across } F_{1}: y_{2}^{\prime}=0,0 \leq y_{3}^{\prime} \leq D_{1}}
\end{aligned}
$$

The resulting boundary value problem, can be solved by using modified form of Green's function technique, developed by Maruyama (1966) and correspondence principles :

$(\bar{u})_{2}(Q)=\int(\bar{u})_{2}(P)\left\{G_{13}^{1}(Q, P) d \xi_{2}-G_{12}^{1}(Q, P) d \xi_{3}\right\}$ where the integration is taken over the fault $F_{1}$ and $Q\left(y_{1}, y_{2}, y_{3}\right)$ is the field point in the half space, not on the fault, and $P\left(\xi_{1}, \xi_{2}, \xi_{3}\right)$ is any point on the fault $F_{1}$ and $(\bar{u})_{2}(P)$ is the discontinuity in $(\bar{u})_{2}$ across $F_{1}$ at the point $\mathrm{P}$ while $G^{1}{ }_{13}(Q, P)$ and $G^{1}{ }_{12}(Q, P)$ are two Green's functions are given by :

and

$$
G^{1}{ }_{13}(Q, P)=\frac{1}{2 \pi}\left[\frac{y_{3}-\xi_{3}}{L^{2}}-\frac{y_{3}+\xi_{3}}{M^{2}}\right]
$$

where,

$$
G^{1}{ }_{12}(Q, P)=\frac{1}{2 \pi}\left[\frac{y_{2}-\xi_{2}}{L^{2}}+\frac{y_{2}-\xi_{2}}{M^{2}}\right]
$$

$$
L^{2}=\left(y_{2}-\xi_{2}\right)^{2}+\left(y_{3}-\xi_{3}\right)^{2}, \quad M^{2}=\left(y_{2}-\xi_{2}\right)^{2}+\left(y_{3}+\xi_{3}\right)^{2}
$$

Now $P\left(\xi_{1}, \xi_{2}, \xi_{3}\right)$ being a point on $F_{1}, 0 \leq \xi_{2} \leq D_{1} \cos \theta_{1}, 0 \leq \xi_{3} \leq D_{1} \sin \theta_{1}$ and $\xi_{2}=\xi_{3} \cot \theta_{1}$. We introduce a change of coordinate axes from $\left(\xi_{1}, \xi_{2}, \xi_{3}\right)$ to $\left(\xi^{\prime}{ }_{1}, \xi^{\prime}{ }_{2}, \xi^{\prime}{ }_{3}\right)$ connected by the relations

$$
\begin{gathered}
\xi_{1}=\xi_{1}^{\prime} \\
\xi_{2}=\xi_{2}^{\prime} \sin \theta_{1}+\xi_{3}^{\prime}{ }_{3} \cos \theta_{1} \\
\xi_{3}=d_{1}-\xi_{2}^{\prime} \cos \theta_{1}+\xi_{3}^{\prime} \sin \theta_{1}
\end{gathered}
$$

so that, $\xi_{2}^{\prime}=0,0 \leq \xi_{3}^{\prime} \leq D_{1}$ on $F_{1}$.

Now from (23)

or,

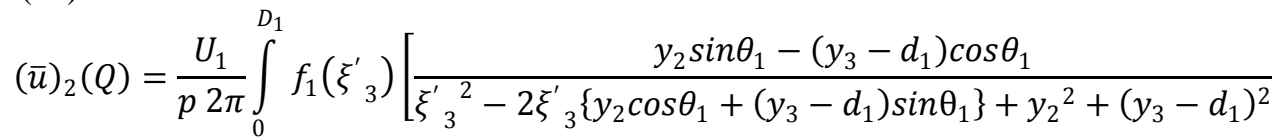

$$
\begin{aligned}
& \left.+\frac{y_{2} \sin \theta_{1}+\left(y_{3}+d_{1}\right) \cos \theta_{1}}{\xi^{\prime}{ }_{3}{ }^{2}-2 \xi^{\prime}{ }_{3}\left\{y_{2} \cos \theta_{1}-\left(y_{3}+d_{1}\right) \sin \theta_{1}\right\}+y_{2}{ }^{2}+\left(y_{3}+d_{1}\right)^{2}}\right] d \xi^{\prime}{ }_{3}
\end{aligned}
$$

where,

$$
(\bar{u})_{2}(Q)=\frac{U_{1}}{p 2 \pi} \psi_{1}\left(y_{2}, y_{3}\right)
$$

$$
\begin{aligned}
& \psi_{1}\left(y_{2}, y_{3}\right)=\int_{0}^{D_{1}} f_{1}\left(\xi_{3}^{\prime}\right)\left[\frac{y_{2} \sin \theta_{1}-\left(y_{3}-d_{1}\right) \cos \theta_{1}}{\xi^{\prime}{ }_{3}^{2}-2 \xi^{\prime}{ }_{3}\left\{y_{2} \cos \theta_{1}+\left(y_{3}-d_{1}\right) \sin \theta_{1}\right\}+y_{2}{ }^{2}+\left(y_{3}-d_{1}\right)^{2}}\right. \\
& \left.+\frac{y_{2} \sin \theta_{1}+\left(y_{3}+d_{1}\right) \cos \theta_{1}}{{\xi^{\prime}}_{3}{ }^{2}-2 \xi^{\prime}{ }_{3}\left\{y_{2} \cos \theta_{1}-\left(y_{3}+d_{1}\right) \sin \theta_{1}\right\}+y_{2}{ }^{2}+\left(y_{3}+d_{1}\right)^{2}}\right] d \xi^{\prime}{ }_{3}
\end{aligned}
$$

Taking Laplace transform with respect to $t_{1}$ and noting that

Now from (15),

$$
\begin{gathered}
(u)_{2}=0 \text { for } t_{1} \leq 0 \\
(u)_{2}=\frac{U_{1}}{2 \pi} \psi_{1}\left(y_{2}, y_{3}\right) H\left(t-T_{1}\right)
\end{gathered}
$$

where,

$$
\begin{gathered}
\left(\overline{\tau_{12}}\right)_{2}=\frac{(\mu+2 \eta p)}{1+\frac{\eta p}{\mu}} \frac{\partial(\bar{u})_{2}}{\partial y_{2}} \\
=\frac{(\mu+2 \eta p)}{1+\frac{\eta p}{\mu}} \frac{U_{1}}{p 2 \pi} \psi_{2}\left(y_{2}, y_{3}\right)
\end{gathered}
$$




$$
\begin{gathered}
\psi_{2}\left(y_{2}, y_{3}\right)=\frac{\partial}{\partial y_{2}}\left\{\psi_{1}\left(y_{2}, y_{3}\right)\right\} \\
=\int_{0}^{D_{1}} f_{1}\left(\xi^{\prime}{ }_{3}\right)\left[\frac{\xi^{\prime}{ }_{3}^{2} \sin \theta_{1}-2 \xi^{\prime}{ }_{3}\left(y_{3}-d_{1}\right)-\left\{y_{2}{ }^{2}-\left(y_{3}-d_{1}\right)^{2}\right\} \sin \theta_{1}+2 y_{2}\left(y_{3}-d_{1}\right) \cos \theta_{1}}{\left[\xi^{\prime}{ }_{3}{ }^{2}-2 \xi^{\prime}{ }_{3}\left\{y_{2} \cos \theta_{1}+\left(y_{3}-d_{1}\right) \sin \theta_{1}\right\}+y_{2}{ }^{2}+\left(y_{3}-d_{1}\right)^{2}\right]^{2}}\right. \\
\left.+\frac{\xi_{3}^{\prime}{ }_{3}{ }^{2} \sin +2 \xi^{\prime}\left(y_{3}+d_{1}\right)-\left\{y_{2}{ }^{2}-\left(y_{3}+d_{1}\right)^{2}\right\} \sin \theta_{1}-2 y_{2}\left(y_{3}+d_{1}\right) \cos \theta_{1}}{\left[\xi^{\prime}{ }_{3}{ }^{2}-2 \xi^{\prime}{ }_{3}\left\{y_{2} \cos \theta_{1}-\left(y_{3}+d_{1}\right) \sin \theta_{1}\right\}+y_{2}{ }^{2}+\left(y_{3}+d_{1}\right)^{2}\right]^{2}}\right] d \xi^{\prime}
\end{gathered}
$$

Now taking Laplace Inverse transformation and noting that

Similarly from (16) we can find out

$$
\begin{gathered}
\left(\tau_{12}\right)_{2}=0 \text { for } t_{1} \leq 0 \\
\left(\tau_{12}\right)_{2}=\frac{\mu U_{1}}{2 \pi} H\left(t-T_{1}\right)\left(1+e^{-\frac{\mu t}{\eta}}\right) \psi_{2}\left(y_{2}, y_{3}\right)
\end{gathered}
$$

where,

$$
\left(\tau_{13}\right)_{2}=\frac{\mu U_{1}}{2 \pi} H\left(t-T_{1}\right)\left(1+e^{-\frac{\mu t}{\eta}}\right) \psi_{3}\left(y_{2}, y_{3}\right)
$$

$$
\begin{gathered}
\psi_{3}\left(y_{2}, y_{3}\right)=-\int_{0}^{D_{1}} f_{1}\left(\xi_{3}^{\prime}\right)\left[\frac{\xi_{3}^{\prime}{ }_{3} \cos \theta_{1}-2 \xi^{\prime}{ }_{3} y_{2}+\left\{y_{2}{ }^{2}-\left(y_{3}-d_{1}\right)^{2}\right\} \cos \theta_{1}+2 y_{2}\left(y_{3}-d_{1}\right) \sin \theta_{1}}{\left[\xi^{\prime}{ }_{3}{ }^{2}-2 \xi^{\prime}{ }_{3}\left\{y_{2} \cos \theta_{1}+\left(y_{3}-d_{1}\right) \sin \theta_{1}\right\}+y_{2}{ }^{2}+\left(y_{3}-d_{1}\right)^{2}\right]^{2}}\right. \\
\left.-\frac{\xi_{3}{ }_{3}{ }^{2} \cos \theta_{1}-2 \xi^{\prime}{ }_{3} y_{2}+\left\{y_{2}{ }^{2}-\left(y_{3}+d_{1}\right)^{2}\right\} \cos \theta_{1}-2 y_{2}\left(y_{3}+d_{1}\right) \sin \theta_{1}}{\left[\xi^{\prime}{ }_{3}{ }^{2}-2 \xi^{\prime}{ }_{3}\left\{y_{2} \cos \theta_{1}-\left(y_{3}+d_{1}\right) \sin \theta_{1}\right\}+y_{2}{ }^{2}+\left(y_{3}+d_{1}\right)^{2}\right]^{2}}\right] d \xi^{\prime}{ }_{3}
\end{gathered}
$$

In the similar way, we can compute $(u)_{3},\left(e_{12}\right)_{3},\left(\tau_{12}\right)_{3},\left(\tau_{13}\right)_{3}$ the displacement, stresses and strains components due to sudden movement of the fault $F_{2}$ by simple linear transformation of coordinates $\left(y_{1}, y_{2}, y_{3}\right)$ and $\left(\xi_{1}^{\prime}, \xi_{2}^{\prime}, \xi_{3}^{\prime}\right)$ to $\left(z_{1}, z_{2}, z_{3}\right)$ and $\left(\eta_{1}^{\prime}, \eta_{2}^{\prime}, \eta_{3}^{\prime}\right)$.

where,

$$
\begin{gathered}
(u)_{3}=\frac{U_{2}}{2 \pi} H\left(t-T_{2}\right) \phi_{1}\left(y_{2}, y_{3}\right) \\
\left(e_{12}\right)_{3}=\frac{U_{2}}{2 \pi} H\left(t-T_{2}\right) \phi_{2}\left(y_{2}, y_{3}\right) \\
\left(\tau_{12}\right)_{3}=\frac{\mu U_{2}}{2 \pi} H\left(t-\mathrm{T}_{2}\right)\left(1+e^{-\frac{\mu t}{\eta}}\right) \phi_{2}\left(y_{2}, y_{3}\right) \\
\left(\tau_{13}\right)_{3}=\frac{\mu U_{2}}{2 \pi} H\left(t-T_{2}\right)\left(1+e^{-\frac{\mu t}{\eta}}\right) \phi_{3}\left(y_{2}, y_{3}\right)
\end{gathered}
$$

$$
\begin{aligned}
& \phi_{1}\left(y_{2}, y_{3}\right)=\int_{0}^{D_{2}} f_{2}\left(\eta^{\prime}{ }_{3}\right)\left[\frac{\left(y_{2}-D\right) \sin \theta_{2}-\left(y_{3}-d_{2}\right) \cos \theta_{2}}{\eta_{3^{\prime}}{ }^{2}-2 \eta^{\prime}{ }_{3}\left\{\left(y_{2}-D\right) \cos \theta_{2}+\left(y_{3}-d_{2}\right) \sin \theta_{2}\right\}+\left(y_{2}-D\right)^{2}+\left(y_{3}-d_{2}\right)^{2}}\right. \\
& \left.+\frac{\left(y_{2}-D\right) \sin \theta_{2}+\left(y_{3}-d_{2}\right) \cos \theta_{2}}{\eta_{3^{\prime}}{ }^{2}-2 \eta^{\prime}{ }_{3}\left\{\left(y_{2}-D\right) \cos \theta_{2}-\left(y_{3}-d_{2}\right) \sin \theta_{2}\right\}+\left(y_{2}-D\right)^{2}+\left(y_{3}-d_{2}\right)^{2}}\right] d \eta^{\prime} \\
& \phi_{2}\left(y_{2}, y_{3}\right) \\
& =\int_{0}^{D_{2}} f_{2}\left(\eta_{3}^{\prime}\right)\left[\frac{\eta_{3}^{\prime}{ }_{3}^{2} \sin \theta_{2}-2 \eta_{3}^{\prime}\left(y_{3}-d_{2}\right)-\left\{\left(y_{2}-D\right)^{2}-\left(y_{3}-d_{2}\right)^{2}\right\} \sin \theta_{2}+2\left(y_{2}-D\right)\left(y_{3}-d_{2}\right) \cos \theta_{2}}{\left[\eta_{3}^{\prime}{ }^{2}-2 \eta^{\prime}\left\{\left(y_{2}-D\right) \cos \theta_{2}+\left(y_{3}-d_{2}\right) \sin \theta_{2}\right\}+\left(y_{2}-D\right)^{2}+\left(y_{3}-d_{2}\right)^{2}\right]^{2}}\right. \\
& \left.+\frac{\eta_{3}^{\prime}{ }^{2} \sin \theta_{2}+2 \eta_{3}^{\prime}\left(y_{3}-d_{2}\right)-\left\{\left(y_{2}-D\right)^{2}-\left(y_{3}-d_{2}\right)^{2}\right\} \sin \theta_{2}-2\left(y_{2}-D\right)\left(y_{3}-d_{2}\right) \cos \theta_{2}}{\left[\eta_{3}^{\prime}{ }^{2}-2 \eta^{\prime}{ }_{3}\left\{\left(y_{2}-D\right) \cos \theta_{2}-\left(y_{3}-d_{2}\right) \sin \theta_{2}\right\}+\left(y_{2}-D\right)^{2}+\left(y_{3}-d_{2}\right)^{2}\right]^{2}}\right] d \eta^{\prime}{ }_{3} \\
& \phi_{3}\left(y_{2}, y_{3}\right)= \\
& =-\int_{0}^{D_{2}} f_{2}\left(\eta^{\prime}{ }_{3}\right)\left[\frac{\eta^{\prime}{ }_{3}^{2} \cos \theta_{2}-2 \eta^{\prime}{ }_{3}\left(y_{2}-D\right)+\left\{\left(y_{2}-D\right)^{2}-\left(y_{3}-d_{2}\right)^{2}\right\} \cos \theta_{2}+2\left(y_{2}-D\right)\left(y_{3}-d_{2}\right) \sin \theta_{2}}{\left[\eta_{3}^{\prime}{ }_{3}{ }^{2}-2 \eta^{\prime}{ }_{3}\left\{\left(y_{2}-D\right) \cos \theta_{2}+\left(y_{3}-d_{2}\right) \sin \theta_{2}\right\}+\left(y_{2}-D\right)^{2}+\left(y_{3}-d_{2}\right)^{2}\right]^{2}}\right.
\end{aligned}
$$

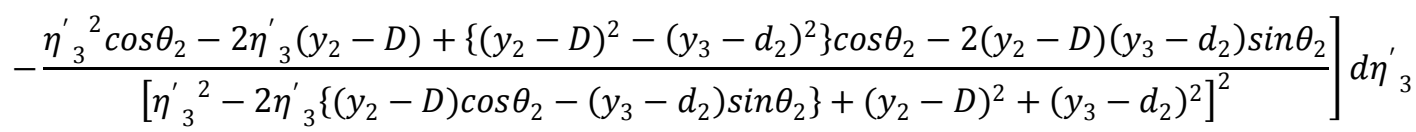




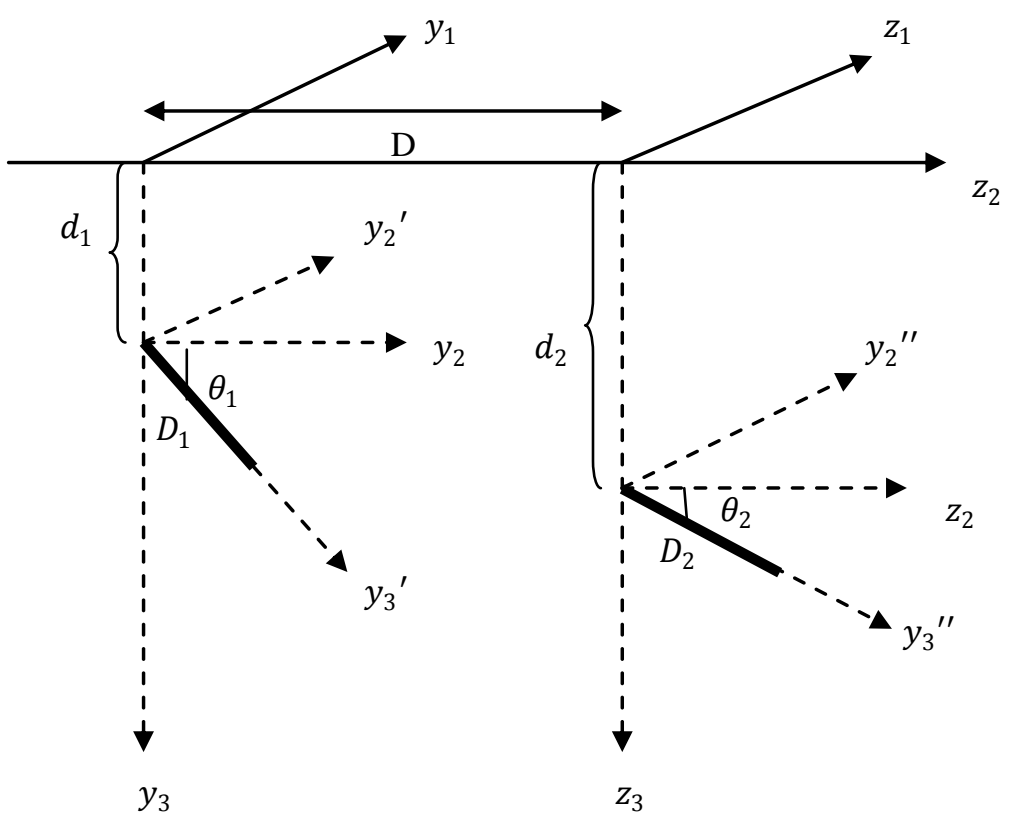

Figure. 1. The section of the fault system by the plane $y_{1}=0$ and relevant coordinate axes

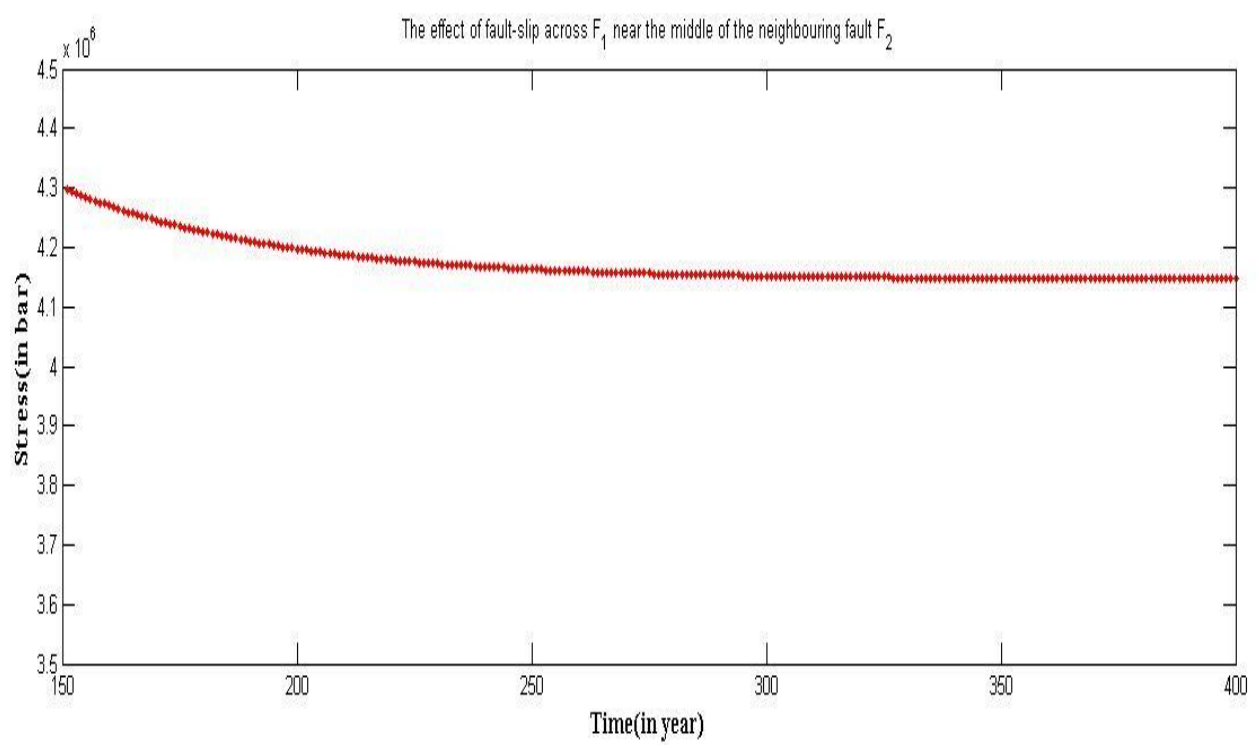

Figure. 2. The effect of fault-slip across $F_{1}$ on the stress accumulation at a point near the middle of the neighbouring fault $F_{2}$ 


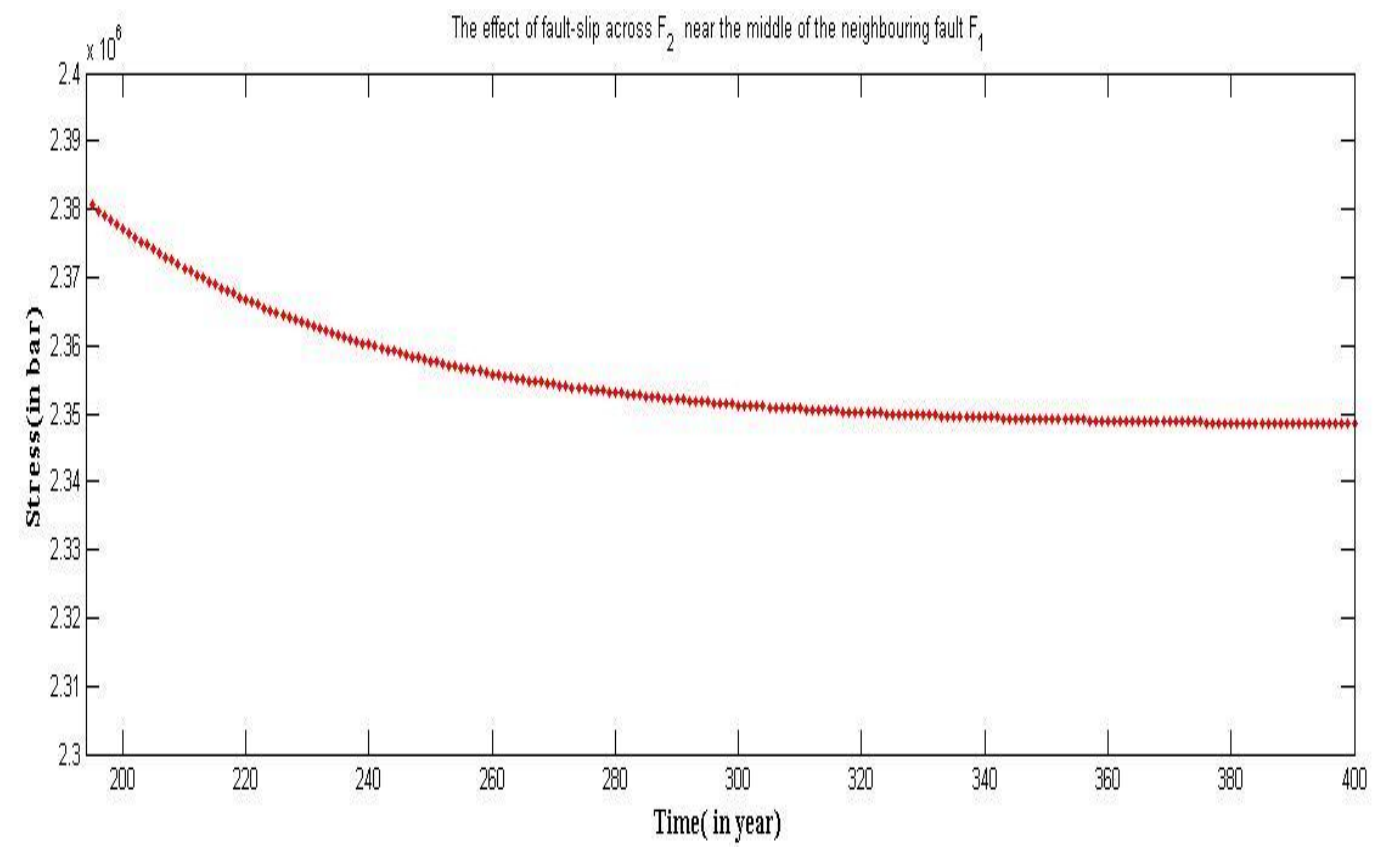

Figure. 3. The effect of fault-slip across $F_{2}$ on the stress accumulation at a point near the middle of the neighbouring fault $F_{1}$

Accumulation against time after the movement of the faults
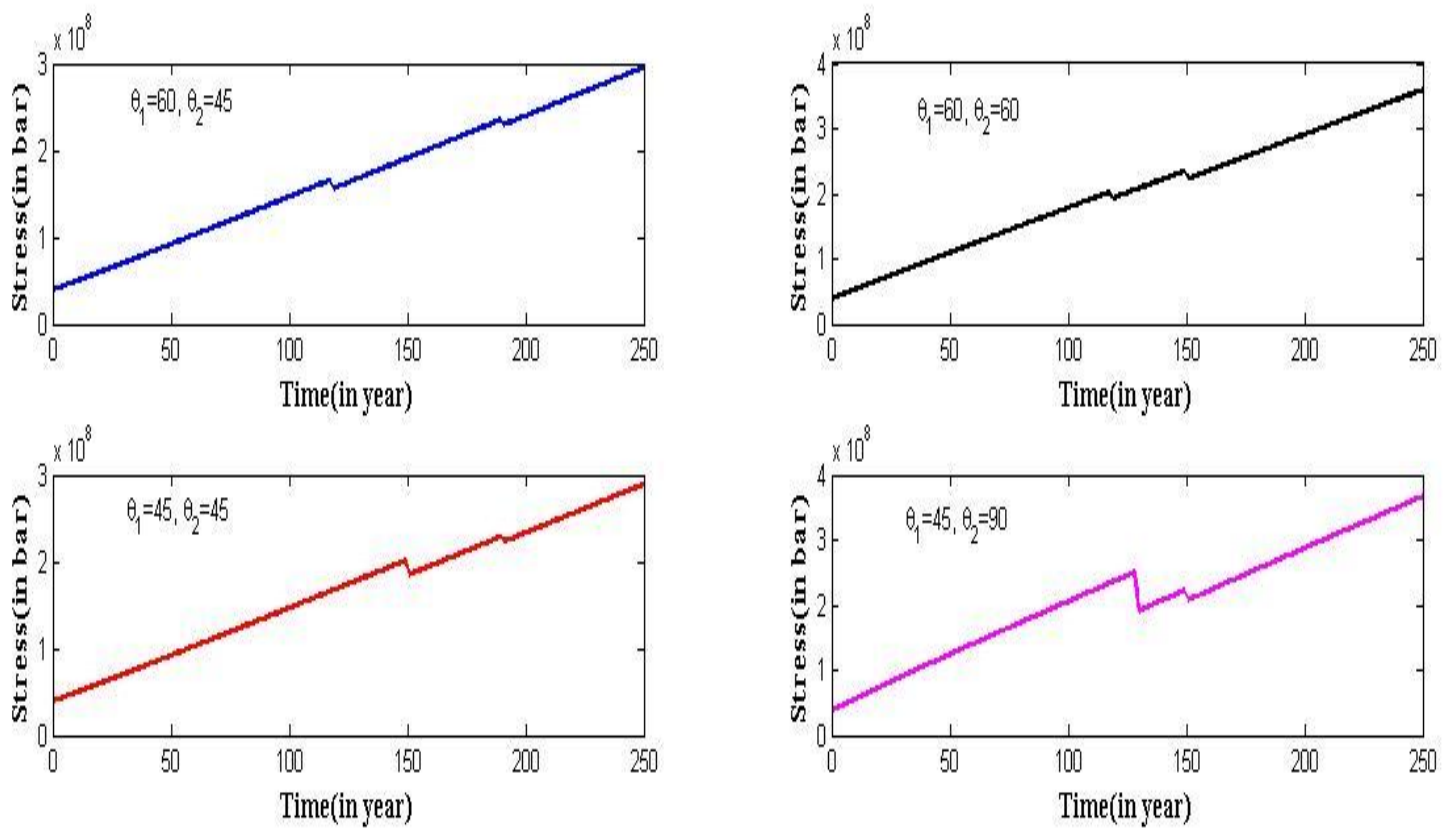

Figure. 4. Stress accumulation against time at a point $y_{2}=8 \mathrm{~km} ., y_{3}=8 \mathrm{~km}$. after the movement of the faults 


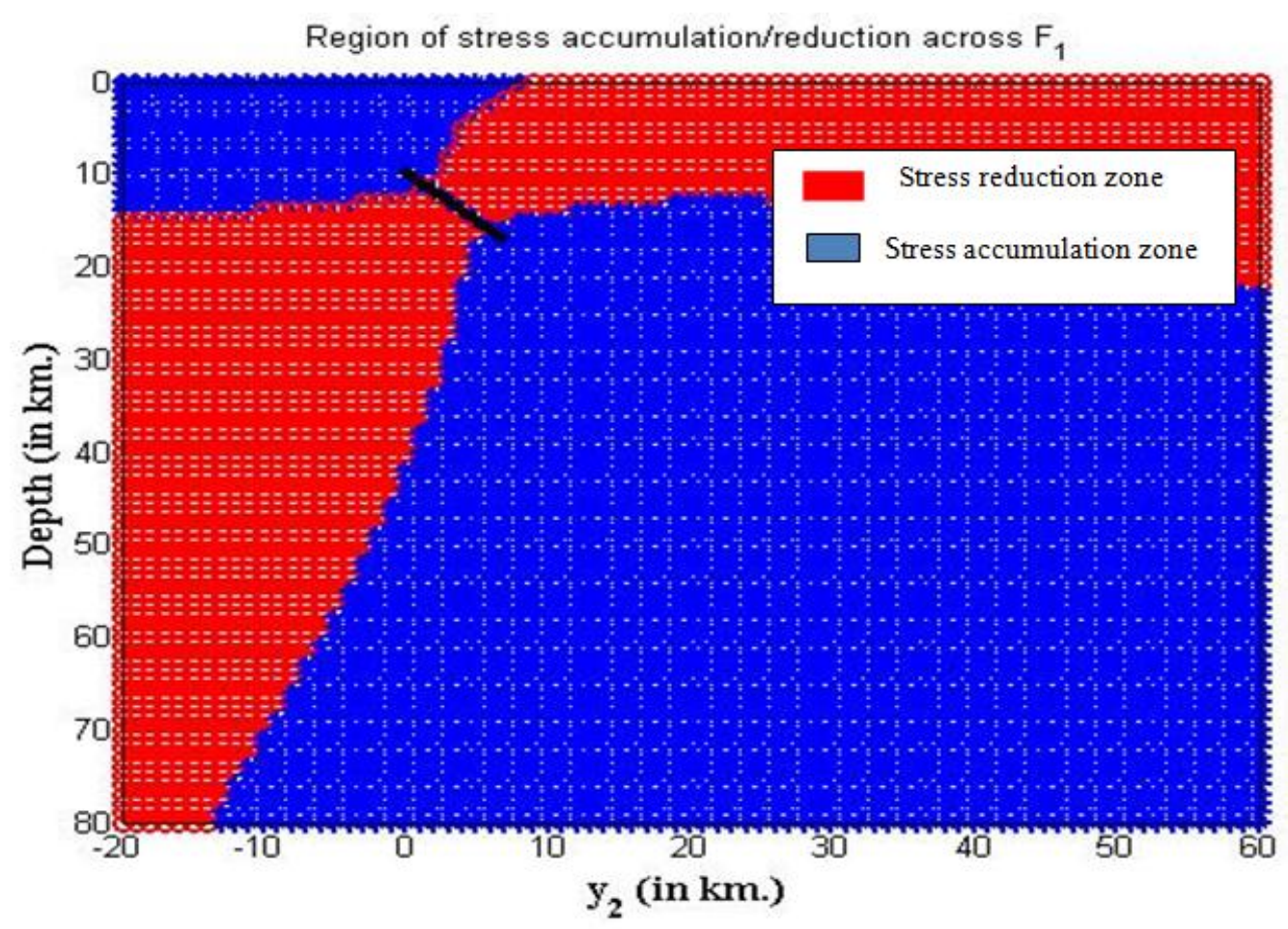

Figure. 5a. Stress accumulation and release due to the fault movement across $F_{1}$ only

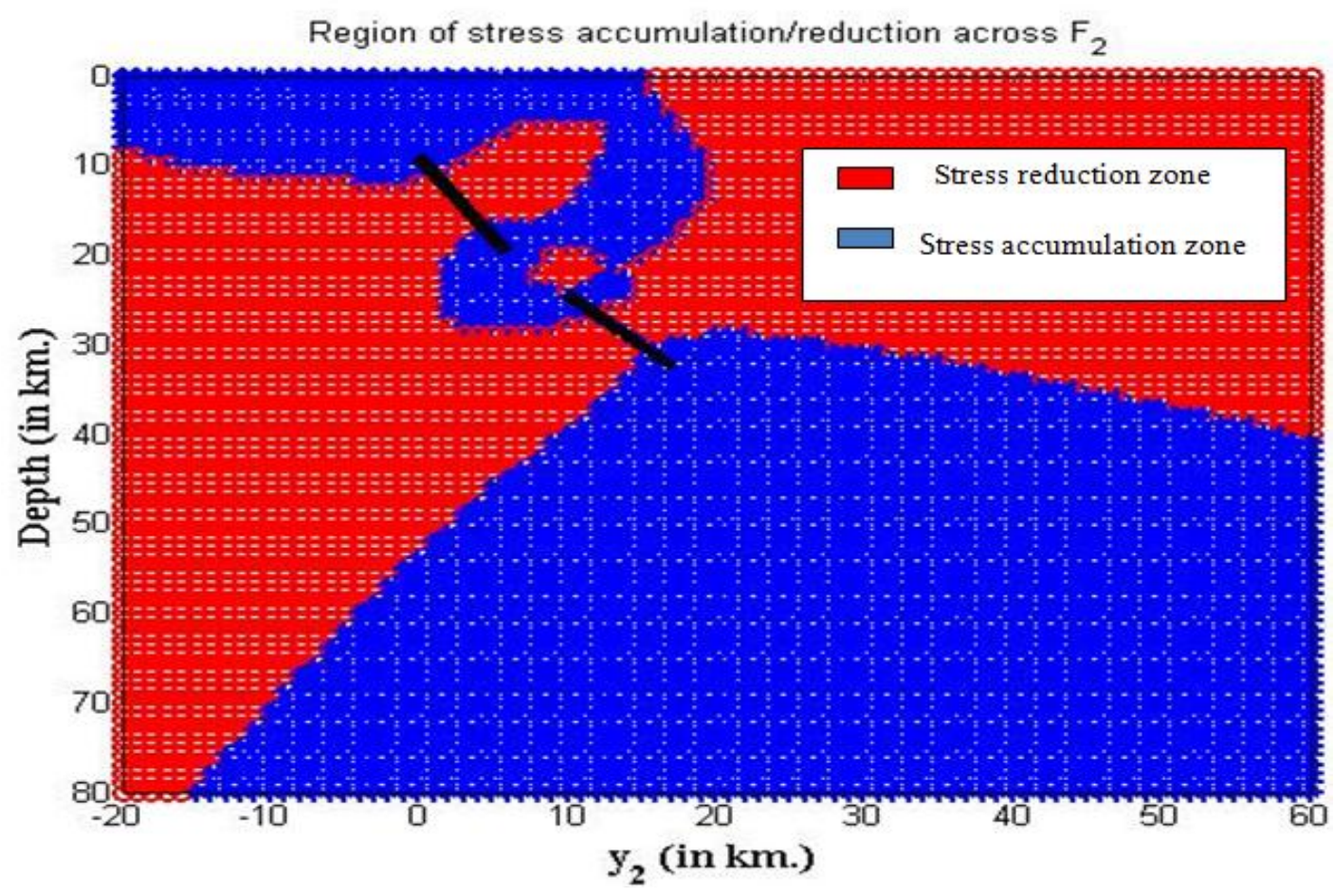

Figure. 5b. Stress accumulation and release after the slip across both the faults $F_{1}$ and $F_{2}$ 


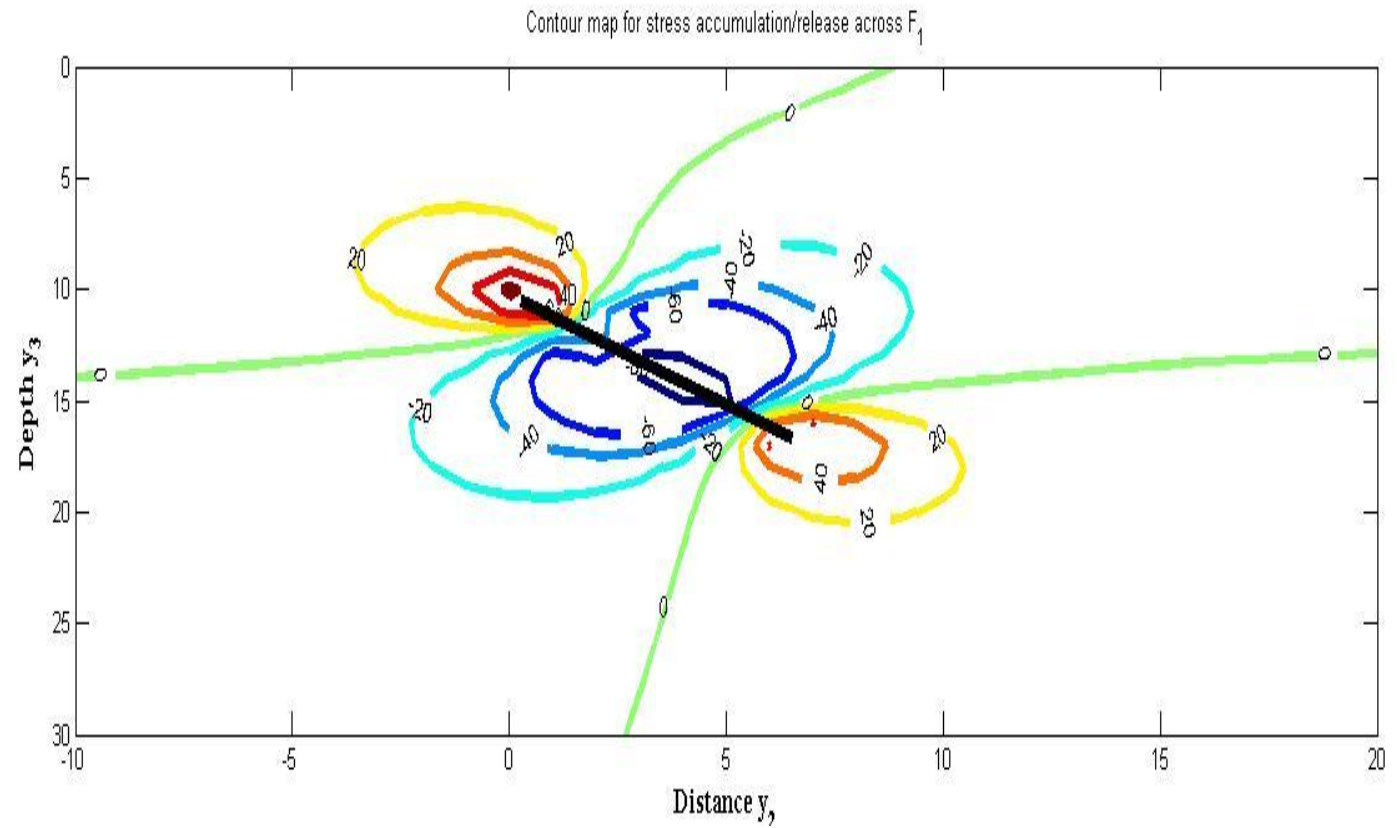

Figure. 6a. Contour map for stress accumulation/release in the medium due to the fault slip across $F_{1}$

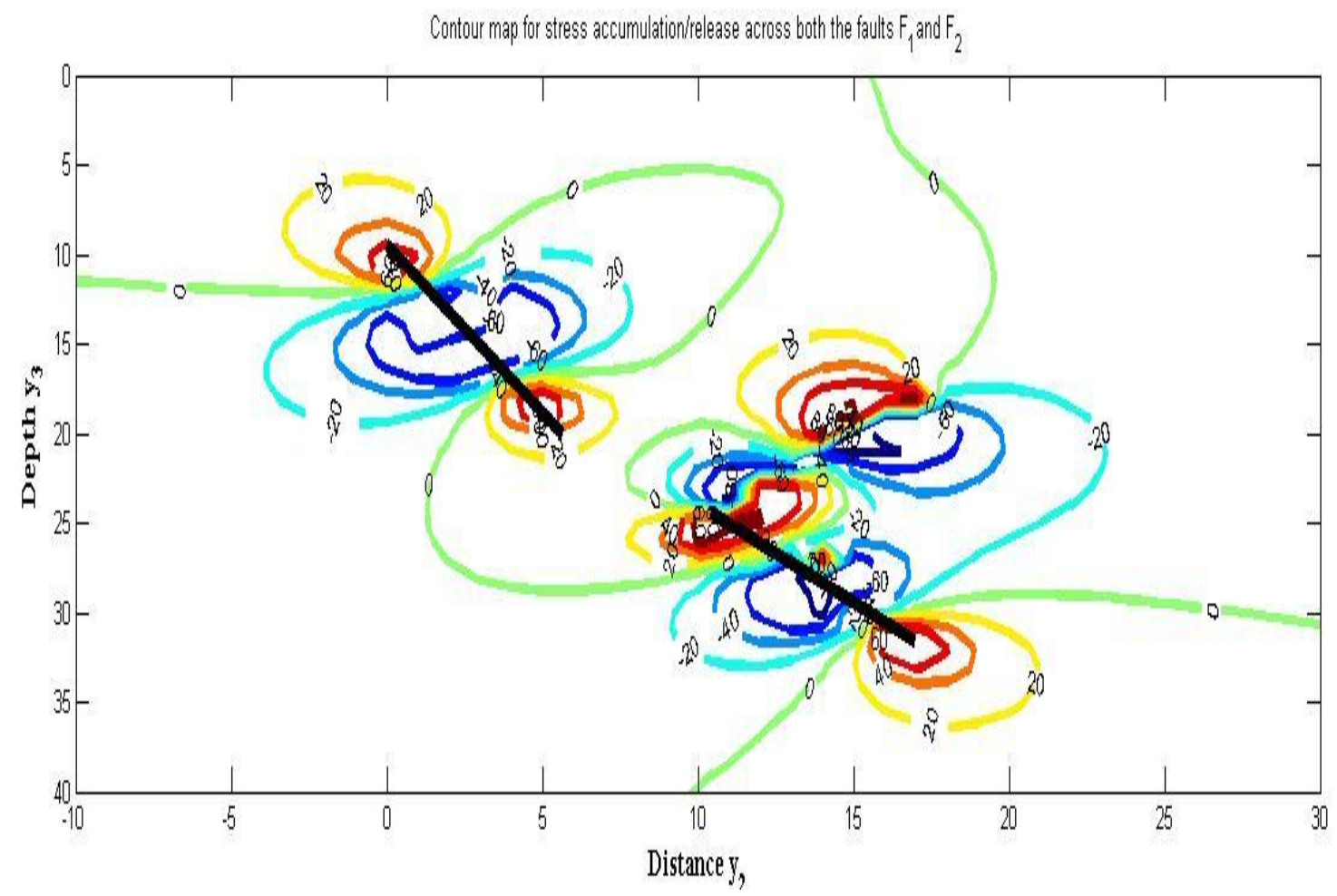

Figure. 6b. Contour map for stress accumulation/release in the medium due to the fault slip across both the faults $F_{1}$ and $F_{2}$ (with faults $F_{1}$ and $F_{2}$ are shown in black colours) 


\section{Acknowledgements}

One of the authors (Papiya Debnath) thanks the Principal and Head of the Department of Basic Science and Humanities, Techno India College of Technology, a unit of Techno India Group (INDIA), for allowing me to pursue the research, and also thanks the Geological Survey of India; Department of Applied Mathematics, University of Calcutta for providing the library facilities.

\section{References}

[1] T. Maruyama, On two dimensional dislocation in an infinite and semi-infinite medium, Bull. earthquake res. inst., tokyo univ., 44, part 3, pp. 811-871, 1966.

[2] K. Rybicki, Static deformation of a multilayered half-space by a very long strike-slip fault, Pure and Applied Geophysics, 110,p1955-1966, 1973.

[3] A. Mukhopadhyay, et.al. On stress accumulation near a continuously slipping fault in a two layer model of lithosphere, Bull. Soc. Earthq. Tech., vol.4, pp. 29-38. (with S.Sen and B. P. Pal), 1980b.17

[4] L.M. Catlhes III, The viscoelasticity of the Earths mantle (Princeton University Press, Princeton, N.J, 1975.15)

[5] K. Aki, P.G. Richards, Quantitive Seismology (University Science Books, Second Ed.,1980)

[6] P. Chift, J. Lin, U. Barcktiausen, Marine and Petroleum Geology (19, 951-970, 2002)

[7] S.I. Karato. (July,2010) Rheology of the Earth's mantle, A historical review Gondwana Research,vol-18, issue-1,pp-17-45. 